\title{
Petabit Photonic Internet
}

\author{
Ryszard S.Romaniuk \\ Faculty of Electronics and Information Technology, Warsaw University of Technology
}

Received April 15, 2011; accepted June 22, 2011; published June 30, 2011

\begin{abstract}
A review of today's understanding of the ultimately broadband photonic Internet is presented. A simple calculation shows a throughput estimate of a core photonic network. The role of photonics is increasing in the development of a physical layer in the future Internet. Photonics leads to better usage of the available bandwidth measured in $\mathrm{b} / \mathrm{s} / \mathrm{Hz}$, increase in the transmission rate from Tbps to Pbps, increase in the transmission distance without signal regeneration in distortion compensated active optical cables, as well as increase in energy/power efficiency measured in W/Gbps. Photonics also leads to fully transparent, low-noise, optical networks and to an increase in bandwidth and network reliability. It is expected that photonics will build a new kind of EM cloud interface between humans and the future global network. The following optical signal multiplexing methods were considered, which are possible without the O/E/O conversion: OTDM, CO-OFDM, OCDM-OCDMA, UDWDM.
\end{abstract}

The photonic Internet comprises a core transport network of ultimate capacity and numerable access networks of large variety and changing capacity - heavily depending on the specific application.

The development of electronic and optoelectronic technologies, microsystems and information technologies, for three decades, in combination with a new psychological and sociological understanding of the global network, has created a stable background for very fast evolutional changes of the Internet [1]. The global network is researched in a much wider context than a mere advanced technical infrastructure [2-7].

It seems now that photonics, together with electronics and mechatronics, will be able to create more than an advanced technical infrastructure, in the form of a completely new kind of interface of psychological and physiological nature. It would be a sort of more direct interface of human senses to the global network. The necessary condition is the existence of a virtual infrastructure of the throughput not confining the applications, including fast, real-time transmission of contents as hi-res 2-D and 3-D images. The 100GbE, close to standardization, has recently been commercialized, and is a proof of these processes.

The next logical step is the $1 \mathrm{~Tb} / \mathrm{s}$ photonic Ethernet. This is an extension to the IEEE 802.3 standard. The backbone network of the future Internet with access links $1 \mathrm{~Tb} / \mathrm{s}$, which is now researched, goes into the throughputs of $100 \mathrm{Tbit} / \mathrm{s}$. The next step is a network of the throughput reaching $400 \mathrm{Tbit} / \mathrm{s}$ and $1 \mathrm{~Pb} / \mathrm{s}$. Today, a stabilized standard for a single fiber in a core network, taking into account the costs, seems to be $10 \mathrm{~Tb} / \mathrm{s}$. The throughput of 1Tbit/s seems also justified economically and technically for major Ethernet access links. Now, the emphasis is put on deeper "photonization" of the Internet. This stems from the development of optical fiber communication and all optical integrated optic processors. This also stems from big costs of manufacturing of high speed electronics, entering the domain of THz.

Electronics is a bottleneck in the domain of digital signal processing at these speeds, which includes the application of advanced modulation schemes of very high spectral efficiency. The fastest commercial DAC/ADC integrated circuits work at a speed of $20 \mathrm{GHz}$. The solution is proper granularity of an electronic bandwidth.

Optoelectronics in the Future Internet: Optoelectronics is expected to address the following issues:

increase the throughput of optical transmission channels, up to Pbps; manage effectively the burst traffic and irregular demand for bandwidth;

build all optical networks of an unconfined bandwidth; build intelligent photonic physical networks with arbitrarily and dynamically configured virtual networks, optimally task oriented;

project a 3D image in free space and create virtual reality for full submergence of a human being and feeling of real presence and participation;

build flexible displays and transparent illuminators integrated with civil engineering infrastructure;

build new generation of personal digital assistants, which are an integrated image interface between an individual and the virtual world;

organize human vicinity as an interface to global network.

The photonic, transparent network does not require intermediate $\mathrm{O} / \mathrm{E} / \mathrm{O}$ signal transformation. Intelligent light paths of different colors are configured dynamically between the relevant users. The paths are opened without collisions in the complex network structure. The network transmits optical signals of arbitrary rate and arbitrary format of modulation, including synchronous and IP. Generation of optimal networks for particular sub-tasks, with reserved relevant transmission and processing resources is done on demand of the user. The users are virtual entities, infrastructure, things, machines, and human beings. Direct usage by humans is much smaller 
than by things. The network is virtual, the physical layer is practically invisible and does not influence its activities.

Today, such a network is tested physically in laboratories on the level of its single components, functional circuits and single links. The tests concern ultimate performance of these components in terms of signal transmission, data aggregation - concentration, multiplexing data processing and acquisition. The rate for a single optical fiber link has recently neared the mark of $100 \mathrm{~Tb} / \mathrm{s}$. The used transmission techniques are concatenated OTDM/DWDM and CO-OFDM/DWDM.

Current understanding of the Future Internet: It is mainly a machine environment. The human Internet consists of three layers: contents, services and social. The Internet of things is mainly the communication of machines (M2MC) and machine interface to the Internet of humans. The Internet of things will occupy from $70 \%$ to $95 \%$ of the network. Things are civilization infrastructure, industry, production, environment monitoring, safety, human vicinity. Machine communication creates massive data streams. Human-human and human-machine communication are data, sound, images and text. The Internet of things will use optical links and transmission networks unconfined in bandwidth. Mobile access networks will serve mobile machines like cars. The stationary reconfigurable networks will serve distributed environmental sensors and RFID devices. The Internet of humans will provide mobile access to the aforementioned three layers via virtual reality, dynamically created on demand, around a person. The role of photonics will be data transmission, generation of 3D images and sounds, signal processing, creation of virtual reality with submerging impression.

The man-machine interface will be more complex and functional. Further development in the submerging technologies will be combined with the development of such tools as: effective on-line 3D imaging, monitoring of human well being (including monitoring and interaction with physiological and psychological parameters), body area network BAN. Simple versions of such networks are now under development for mobile remote-medical purposes. The surface of a human body will be used for intelligent interaction with the network. Such interaction may evolve as a sort of additional artificial human sense. It may include the generation of images directly on the retina, but the extent of these technologies is broader. Some of these techniques may not be very safe, and arouse a lot of anxiety.

The development of avatars involves a kind of social acceptance, or redefining the role of individuals, personal autonomy and freedom as well as society. This perspective is increasingly clearly visible today. The role of optoelectronics is sensors, measurement techniques, image communication, and much more - encircling a human being with an intelligent EM cloud. This optical cloud, modulated by real and virtual senses, also by physiology and psychology will be an interface between an individual, one's organism and the global network.

Petabit Internet: Let us assume the following technical data of a photonic link of the future Internet: transmission system with WDM-CO-OFDM multiplexing; optical fiber bandwidth used is inside the loss window $L<0.5 \mathrm{~dB} / \mathrm{km}$ with the usage efficiency of $90 \%$ (natural spectral efficiency of WDM) or, approximately, equal to $\Delta \lambda \approx 400 \mathrm{~nm}$; equivalent natural bandwidth in the frequency domain is $B=50 \mathrm{THz}$; spectral efficiency of transmission (today attainable with difficulty in optical domain, but available in the RF domain and used in cellular telephony systems) of the order $E=10 \mathrm{~b} / \mathrm{s} / \mathrm{Hz}$; easily obtainable polarization multiplexing 1:2 with acceptable SNR values. Using these data, the following throughputs are obtained: around $1 \mathrm{Pbit} / \mathrm{s}$ for trunk optical fiber systems and 2-3Pbit/s for local area network systems. Assuming an optical fiber cable between very large IX of the future Internet containing 100 lighten and 100 dark fibers in each direction, for full duplex transmission, the throughput is $100 \mathrm{Pbit} / \mathrm{s}$. This data rate is five orders of magnitude faster than the planned development of the standardized, guaranteed throughput equal to $1 \mathrm{Tbit} / \mathrm{s}$ offered today for the core network of the photonic Internet.

Shannon-Hartley law: For colored noise, the S-H law is:

$$
\begin{aligned}
& C=\int_{0}^{B} \log _{2}\left(1+\frac{S(f)}{N(f)}\right) d f \\
& \mathrm{C}[\mathrm{b} / \mathrm{s}] \underset{\mathrm{N}[\mathrm{W}]}{ }
\end{aligned}
$$

where: $C$ - channel capacity, $B$ - bandwidth, $S$ - output signal power, $N=g B$ - noise power, $f$ - frequency, $g$ - noise spectral density, $R[\mathrm{~b} / \mathrm{s}]$ - line rate (throughput), $\mathrm{SNR}[\mathrm{dB}]=10 \log _{10}(\mathrm{~S} / \mathrm{N})$.

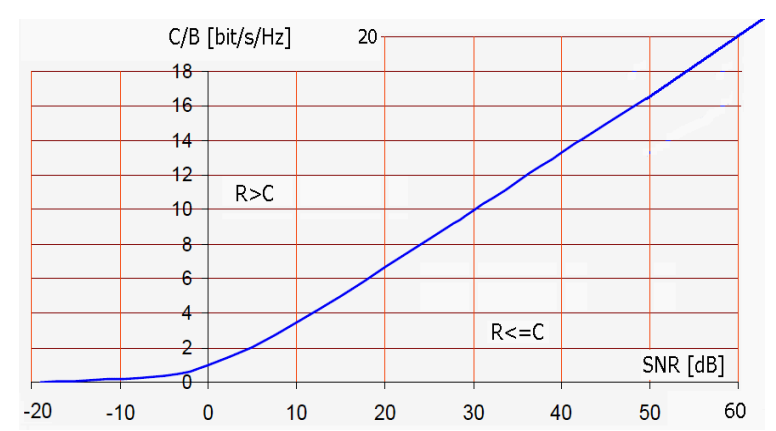

Fig. 1. Shannon-Hartley diagram: spectral efficiency C/B of (photonic) transmission vs. signal to noise ratio. Function is also presented for bit energy $E_{b}=S / C[W / b / s]$, or $E_{b} / g[d B]$ 
For AWGN noise $\mathrm{C}=B \log _{2}(1+S / N)$. Two approximations hold: $C=0.33 B(\mathrm{SNR}[\mathrm{dB}])$ for $S / N>>1$ and $C=1.44 B(S / N)=1.44 g S$ for $S / N<<1$, as shown in Fig. 1 .

Assuming a low-noise channel $\mathrm{SNR}=30 \mathrm{~dB}$ for trunk photonic transmission and $B=50 \mathrm{THz}$, one obtains $C=0.5 \mathrm{~Pb} / \mathrm{s}$ and the spectral efficiency $C / B=10 \mathrm{~b} / \mathrm{s} / \mathrm{Hz}$. For a noisy photonic channel, the transmission does not depend on the bandwidth, and the solutions are LDPC or Turbo-FEC codes.

Next Generation UDWDM: WDM has gone a long distance during the two decades. The bandwidth reserved for a single channel is: CWDM-20nm, WDM-0.8nm (100GHz), DWDM-50/25GHz, UDWDM- 6/3GHz, NGUDWDM 1.5/075GHz. Assuming $L<2.0 \mathrm{~dB} / \mathrm{km}$, $\Delta \lambda \approx 1.2 \mu \mathrm{m}$, one can accommodate 64 channels in $100 \mathrm{GHz}$, see Fig. 2. The optical band has $1200 \mathrm{~nm}: 0.8 \mathrm{~nm}=1500$ channels. UDWDM system capacity is: $1500 * 64=96000$ colors with separation of $1.5 \mathrm{GHz}$. Assuming $1 \mathrm{~Gb} / \mathrm{s} /$ color, the aggregated traffic reaches $100 \mathrm{~Tb} / \mathrm{s}$. Assuming 5Gb/s/color and adding 2:1 PDM we obtain the same value of $1 \mathrm{~Pb} / \mathrm{s}$ for ultimate throughput.

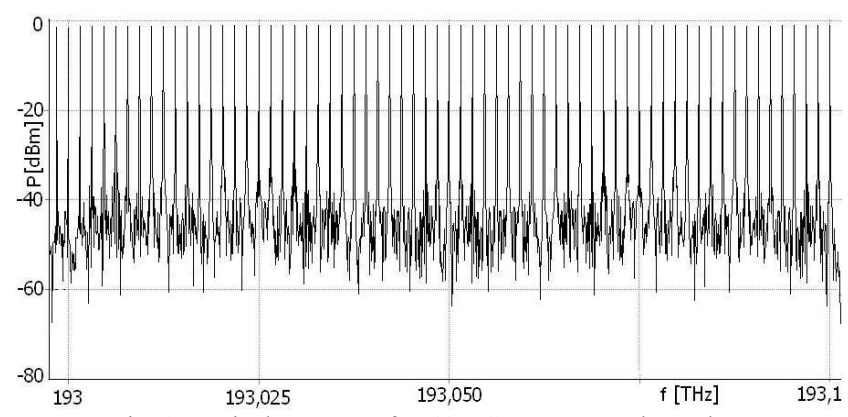

Fig. 2. Optical spectrum for 64 NG-UDWDM channels: 100GHz:64=1.5625GHz.

Existing example: The DWDM system shown by NTT during the OFC2010 has the following parameters: $R \approx 70 \mathrm{~Tb} / \mathrm{s}$, modulation $16 \mathrm{QAM}$ with $2 \mathrm{PDM}$, band 1527 $1620 \mathrm{~nm}, D=25 \mathrm{GHz}, E=6.4 \mathrm{~b} / \mathrm{s} / \mathrm{Hz}$. Electronics worked at an acceptably low rate of $>20$ Gbaud. Squeezing the $25 \mathrm{GHz}$ standard to $1.5 \mathrm{GHz}$, or interleaving the colors 16 times, one obtains $70 \mathrm{~Tb} / \mathrm{s} * 16 \approx 1.1 \mathrm{~Tb} / \mathrm{s}$.

Modulation schemes in the Photonic Internet: The multiplexing methods used for optical fiber transmission systems are [1-7]:

WDM and dense standards DWDM, UDWDM with direct detection and intensity modulation (IM DD), coherent WDM - single carrier, orthogonal WDM-SC, fully optical OFDM, with direct detection, single carrier, (O-OFDM and CO-OFDM), coherent optical OFDM, CO-OFDM), OFDM with electro-optically modulated subcarriers, with direct detection, single carrier, multiband MB-OFDM, with a single band in single time, and multi-band version,

OFDM with non-orthogonal direct sub-carrier multiplexing,

band multiplexing BM-OFDM with non-orthogonal splitting to sub-bands,

orthogonal band multiplexing OBM-OFDM with orthogonal splitting to sub-bands, multiple carriers, hybrid optoelectronic method of relatively small granularity of electronics, due to splitting of the whole band to narrower sub-bands which, in turn, are subject to easy and cheap $\mathrm{O} / \mathrm{E}$ conversion.

The multiplexing methods are subject to concatenation. Electronic or optoelectronic methods are joined with optical. In the future, all optical methods will be concatenated. The following methods are added: TDMWDM and OTDM-WDM, OFDM-WDM, and OCDMWDM.

Polish perspective [8-10]: The daily statistics of DE-CIX (Frankfurt, April 2011) display peak traffic around 2Tb/s. Similar traffic is at LINX (London). PLIX (Warsaw) shows traffic around $120 \mathrm{~Gb} / \mathrm{s}$. Big PLIX participants own $40 \mathrm{~Gb} / \mathrm{s}$ and $10 \mathrm{~Gb} / \mathrm{s}$ ports. The best ISP in the Warsaw area provides $120 \mathrm{Mb} / \mathrm{s}$ via cable/FTTH for private users.

In conclusion, optoelectronics offers now high spectral efficiency of digital transmission, over $5 \mathrm{~b} / \mathrm{s} / \mathrm{Hz}$. This allows us not to give up a very high rate of elementary data streams, equal to $100 \mathrm{Gbps}$, at the granularity of electronic bandwidth $20 \mathrm{~Gb} / \mathrm{s}$. This granularity of electronics is obtained now at a low cost. Splitting to subbands is performed by anti-aliasing filters. A single ADC circuit of $20 \mathrm{GS} / \mathrm{s}$ is satisfactory to receive a $100 \mathrm{~Gb} / \mathrm{s}$ OFDM signal. Direct work with 100Gbps electronics increases the system costs. Such systems are researched in laboratory conditions, to replace 20 and $40 \mathrm{GHz}$ granularity with $100 \mathrm{GHz}$ in the future. Internet 2025 , according to today's predictions, will carry several tens of $\mathrm{Pb} / \mathrm{s}$ of aggregated traffic (three orders of magnitude more than today), will need several tens of TW of power and will employ tens of millions of us.

\section{References}

[1] R.S. Romaniuk, Proc. SPIE 8010, 801003 (2011).

[2] R.S. Romaniuk, Proc. SPIE 8010, 801004 (2011).

[3] R.S. Romaniuk, Proc. SPIE 8010, 801005 (2011).

[4] A. Kalicki, R. Romaniuk, Proc. SPIE 7124, 712410 (2008).

[5] R.S. Romaniuk, Proc. SPIE 6347, 63470Z (2006).

[6] R.S. Romaniuk, Proc.SPIE 5484, 19 (2004).

[7] R.S. Romanuk, Proc. SPIE 5125, 17 (2002).

[8] R.S. Romaniuk, Proc. SPIE 5125, 5 (2002).

[9] W. Wojcik, R.S. Romaniuk, Proc. SPIE 7745, 774508 (2010).

[10] J. Modelski, R.S. Romaniuk, Proc. SPIE 7745, 04, 05, 06 (2010). 